\title{
Toward a generalized friction controller: from the bowed string to unusual musical instruments.
}

\author{
Stefania Serafin \\ Medialogy \\ Aalborg University \\ Denmark \\ sts@cs.aue.auc.dk
}

\author{
Diana Young \\ MIT \\ Media Lab \\ Cambridge, MA \\ diana@media.mit.edu
}

\begin{abstract}
We present case studies of unusual instruments that share the same excitation mechanism as that of the bowed string. The musical saw, Tibetan singing bow, glass harmonica, and bowed cymbal all produce sound by rubbing a hard object on the surface of the instrument. For each, we discuss the design of its physical model and present a means for expressively controlling it. Finally, we propose a new kind of generalized friction controller to be used in all these examples.
\end{abstract}

\section{INTRODUCTION}

Playability of physical models is an area of increasing interest in recent years. As the synthesis techniques have become suitably refined to compare favorably with their real instrument counterparts, access to the many musical possibilities they offer has remained somewhat limited from the standpoint of the performer. That is, though they provide ample material for use in new compositions [8], and even inspire the creation of new compositional techniques and notations [1], they are still generally quite removed from the easy intuitive control of performers. Only when there are natural and instinctive physical ways of playing the sophisticated models in existence today, will these models emerge as true instruments in spite of their virtual origin.

Previously, an ongoing virtual violin project was described $[10,14]$, in which a bowed string controller is used to explore the playability of a violin physical model. Though the violin is an example of a highly sophisticated traditional instrument that requires a very complicated playing technique, it relies on an excitation mechanism that is common to many instruments that may be considered unusual by comparison.

This paper addresses the issues of controlling physical models of instruments that possess a friction-based excitation mechanism such as that found in the bow-string interaction and the musical possibilities such control might afford. Discussed below are the musical saw, Tibetan singing bowl, glass harmonica, and bowed cymbal.

We also propose a new generalized controller for friction driven instruments. This controller is able to drive all the instruments described in this paper, as well as other sonorities which are produced by rubbing dry surfaces.

\section{TIBETAN BOWL AND GLASS HARMON- ICA}

\subsection{The Tibetan bowl}

Oral tradition dates the singing bowl back to $560-180$ B.C. in Tibet. These bowls have been found in temples, monasteries, and meditation halls throughout the world. Singing bowls are said to be made out of five to seven metals such as gold, silver, mercury, copper, iron, metal and tin, each representing a celestial body. Each of these metals is said to produce an individual sound, including partials, and together these sounds produce the exceptional singing sound of the bowl. Each bowl is hand hammered round to produce beautiful harmonic tones and vibrations. Today they are used in music, relaxation, meditation, and healing.

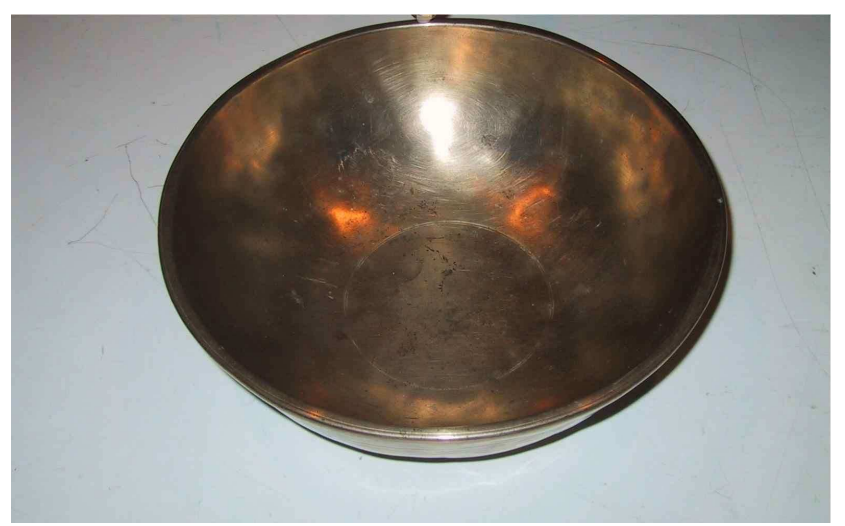

Figure 1: The Tibetan singing bowl used as a starting point for the physical model.

\subsection{The glass harmonica}

Glass harmonicas are musical instruments of two kinds. The first one, invented by Benjamin Franklin, adopts glass bowls turned by a horizontal axle so that one side of the bowl dips into a trough of water. The second one is a combination of wineglasses similar to the ones shown in figure 


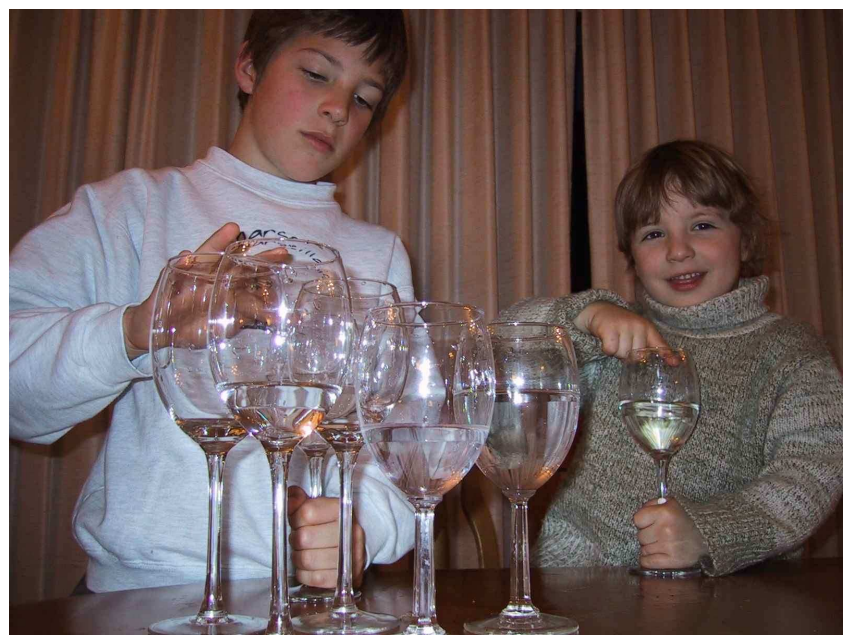

Figure 2: Young performers playing the glass harmonica.

2. Different melodies can be played on a set of tuned glasses (filled with appropriate amounts of water or carefully selected by size), simply by rubbing the edge of the glass with a moist finger. Rubbing rims of glasses in order to produce music became very popular in Europe during the 18th century. Music on glasses has been successfully composed by Mozart, Beethoven, and many others.

\subsubsection{Modeling a Tibetan bowl and a glass harmon- ica}

In [7] we proposed a physical model of a Tibetan bowl implemented using banded waveguides [2]. Banded waveguides are a particular case of waveguide networks in which each waveguide represents one strong resonance of the system. For a detailed description of banded waveguides, see [3]. The strong characteristic beatings of the instrument were implemented using detuned banded waveguides [9]. Considering the similarity of the structure of the Tibetan bowl and the glass harmonica, also the glass harmonica can be modeleled using banded waveguides. The two instruments are driven by the friction driven mechanism described in [5].

\subsection{A Controller for Tibetan bowl and Glass Harmonica}

The physical input parameters that control the excitation of a singing bowl (when bowed/rubbed with the playing stick) are the pressure of the stick against the surface of the bowl and the speed with which the stick travels around the bowl's edge. (The other features that affect the resonance of this instrument are related to the size, shape, and material qualities.)

In order to play a model of a singing bowl, a controller was developed called the HyperPuja [13]. This interface was designed with minimal electronics, including a wireless $R F$ tranmitter, embedded inside the core of a traditional playing stick. The speed of the stick is measured using a small set of magnets adhered (not permanently) to the inside of a traditional Tibetan bowl and Hall sensors on the inside of the stick. (The time between peaks in the output of the Hall sensor indicated the speed with which the stick traverses the rim of the bowl.) Morevoer accelerometers on the bowl stick for added control are inserted.
The pressure of the stick against the bowl was captured by a custom-made force sensitive resistor. The outside of the stick was wrapped in copper foil, over which was placed layer of thin conductive rubber, topped with a layer of copper mesh. The rubber is seen as a resistor, whose resistance decreases with increasing pressure. The sensor assembly was covered using a piece of chamois, similar to the thickness and texture of suede commonly used to wrap playing sticks.

The implementation of this controller allows for easy control of Tibetan bowl models using normal playing techiques. In addition, because the enhancement of the traditional instrument does not significantly impede its acoustic capabilities, it allows for electroacoustic performances combining the virtual bowl sound with the real bowl sound.

Beause of the great similarity between the glass harmonica and the Tibetan singing bowl models, we assert that the same controller may be used to play both.

\section{MUSICAL SAW AND BOWED CYMBAL}

\subsection{The musical saw}

When an ordinary handsaw is bent into an S-shape, an interesting acoustical effect can occur. Tapping the blade of the saw reveals that beyond a certain critical degree of curvature, a very lightly damped vibration mode appears which is confined to the middle region of the $\mathrm{S}$. This confined mode can be excited by a violin bow, to produce the pure sound of the "musical saw".

The origins of the musical saw go back to the early 20th century, especially among the folk instrument community. Some important contributors to the development of the musical saw have been Leon and June Weaver. They also started playing the saw using a violin bow in a lap style, as shown in figure 3 .

\subsubsection{Modeling a musical saw}

In [7] we proposed a physical model of a musical saw implemented using banded waveguides [2]. Given the simplicity of the spectrum of the musical saw, a single banded waveguide can be used to obtain a realistic simulation of the instrument.

\subsection{Bowed cymbals}

As described in [4], the vibration of a cymbal is very similar to the vibration of flat circular plates. While modes are clearly distinguishable at low frequencies, at high frequencies they often mix with one another.

The nonlinear coupling between vibrational modes, moreover, is pretty strong, which makes many partials appear quickly in the spectrum. This is true no matter how the cymbal is excited.

In [4] an investigation of nonlinearities in cymbals is described. The results of exciting a cymbal with a sinusoidal shaker show that, while at low frequency the radiated sound is concentrated at the fundamental of the exciting frequency, increasing the amplitude increases also the relative levels of all the partials. At a critical excitation amplitude the spectrum develops a complete set of subharmonics, and transitions to fully chaotic behavior can appear. The mathematical problem of analyzing cymbal behavior in detail is rather complex. The frequency response of a bowed cymbal presents a large number of potentially active modes. 


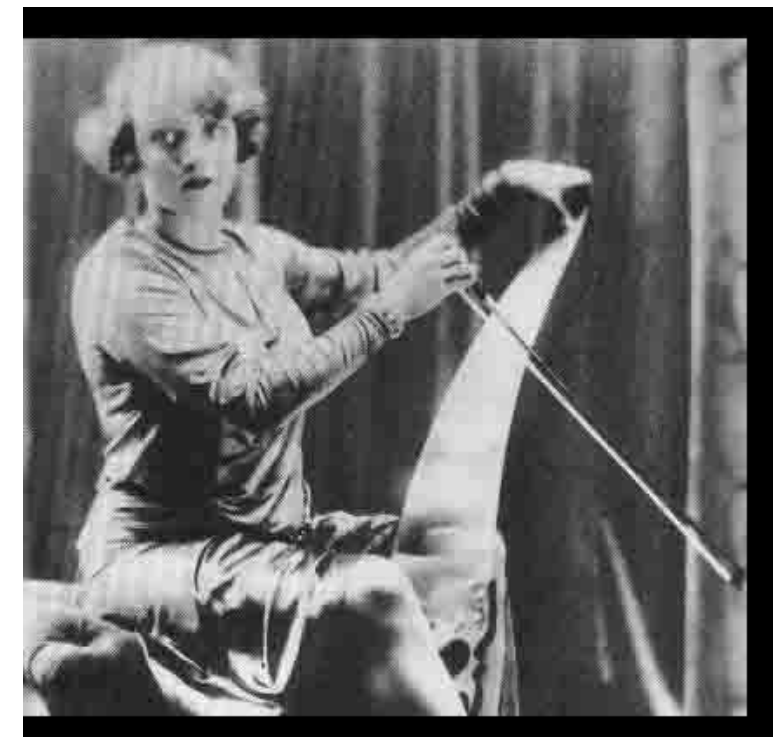

Figure 3: The musical saw.

\subsubsection{Modeling bowed cymbal}

In order to simulate bowed cymbals and plates, in [6] we proposed a structure called banded waveguide mesh. The banded waveguide mesh is an extension to multiple dimensions of the banded waveguide in order to allow a real-time implementation. Low frequency modes are simulated using banded waveguides, while high frequency modes are simulated using a two dimensional waveguide mesh [11].

\subsection{A Controller for musical saw and bowed cymbal}

As with the Tibetan singing bowl, we sought a controller for the musical saw and bowed cymbal that is as traditional in feel and appearance as possible. In order to play these models we used the bow controller as for the bowed string experiments in other recent work.

Primarily, we use the bow controller's capabilities of reflecting speed, using an electric field sensing technique, and force (as seen in the bending of the bow stick) as input parameters to the models. Like the other controller discussed, the implementation of the sensing elements here was done with great care to maintain playability and allow for typical playing techniques and styles.

Again, because the enhancements of the original acoustic interface do not impede the acoustic behaviours of the instrument, it is possible to provide performers with the ability to access both the real and the virtual instruments in the same performance, using the same interface.

For a detailed description of the bow controller, see [12].

\section{TOWARD A GENERALIZED FRICTION CONTROLLER}

Though the two controllers discussed here offer many possibilities to performers due to their traditional feel, appearance, and function, it is legitimate to ask whether in many scenarios a less traditional interface may be more beneficial. Specifically, we are developing a general interface for the control of all friction-driven instrument models.
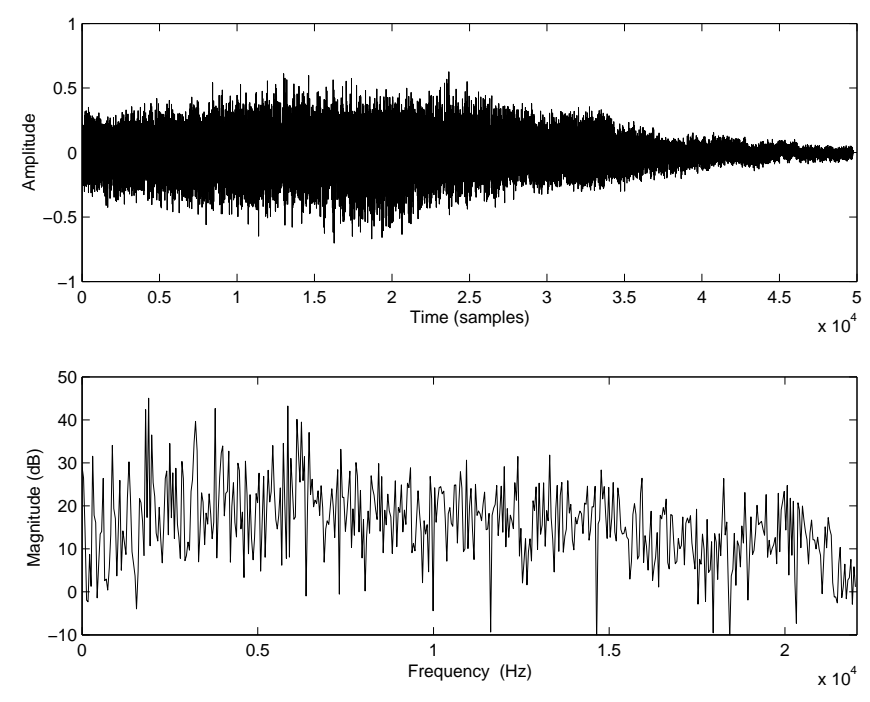

Figure 4: Time and frequency domain representation of a bowed cymbal. Notice the rich spectrum

Such a device would likely share some of the physical qualities of a bow and playing stick, but having characteristics that imply different playing techniques. The challenges on the design of this device are due to the fact that friction sounds are produced in different ways such as perpendicular motion of the exciter on the resonator (like in the case of the bowed string and the musical saw), or circular motion (like in the Tibetan bowl and glass harmonica). We are currently experimenting with having a pressure sensor, a combination of different types of position sensors (Hall technique), strain/bend sensors like the ones used in the bow controller, accelerometers, and gyros for angular velocity.

Such an instrument, however, will give composers and performers great possibilities to explore and extend the sound of friction.

\section{CONCLUSION}

We have presented several case studies of musical instruments that operate by means of a friction-based excitation mechanism much like that of a violin. We have described modelling techniques used to create their virtual instrument counterparts, as well as controllers that may be used to allow intuitive performances of them. Finally, we propose the creation of a generalized friction controller that could be used not only to play all of the virtual instruments highlighted in this paper, but also assist in the creation of hybrid electroacoustic instruments that utilize real-time physical models.

\section{REFERENCES}

[1] M. Burtner. Composing for the (dis)embodied ensemble: Notational systems in (dis)appearances. In Proc. NIME, Montreal, Quebec, May 2003.

[2] G. Essl and P. R. Cook. Banded Waveguides: Towards Physical Modeling of Bowed Bar Percussion Instruments. In Proceedings of the International Computer Music Conference (ICMC), pages 321-324, Beijing, China, 1999. International Computer Music Association (ICMA). 
[3] G. Essl, S. Serafin, P. Cook, and J. O. Smith. Theory of Banded Waveguides. Computer Music Journal, 28(1), 2004.

[4] N. Fletcher. Nonlinearities and the Sound of Musical Instruments. In Acoustical Society of America, 1996.

[5] S. Serafin. The sound of friction: real-time models, playability and musical applications. $\mathrm{PhD}$ thesis, Stanford University, Stanford, CA, 2004.

[6] S. Serafin, P. Huang, and J. O. Smith. The Banded Digital Waveguide Mesh. In Proc. Workshop on Future Directions of Computer Music (Mosart-01), Barcelona, Spain, November 2001.

[7] S. Serafin, P. Huang, S. Ystad, C. Chafe, and S. J. Analysis and synthesis of unusual friction driven musical instruments. In Proceedings of the International Computer Music Conference (ICMC-02), Gotheborg, 2002.

[8] S. Serafin and J. Kojs. The voice of the dragon: a physical model of a rotating corrugated tube. In Proc. DAFX 2003, London, UK, 2003.

[9] S. Serafin, C. Wilkerson, and J. O. Smith. Modeling Bowl Resonators Using Circular Waveguide Networks. In Proceedings of the COST G-6 Conference on Digital Audio Effects (DAFX-02), Hamburg, Germany, 2002.

[10] S. Serafin and D. Young. Bowed string physical model validation through use of a bow controller and examination of bow strokes. In Proc. Stockholm Musical Acoustics Meeting (SMAC), 2003.

[11] S. van Duyne and J. O. Smith. Physical Modeling With the 2D Waveguide Mesh. In Proceedings of the International Computer Music Conference (ICMC), pages 40-47, The Hague, 1993. International Computer Music Association (ICMA).

[12] D. Young. The hyperbow controller: Real-time dynamics measurement of violin performance. In Proc. NIME, 2002.

[13] D. Young and G. Essl. Hyperpuja: A tibetan singing bowl controller. In Proc. NIME, 2003.

[14] D. Young and S. Serafin. Playability evaluation of a virtual bowed string instrument. In Proc. NIME, Montreal, Quebec, May 2003. 